\title{
Efeito do Desbaste e da Qualidade do Sítio na Relação Hipsométrica de Pinus taeda
}

\author{
Hassan Camil David ${ }^{1}$, Julio Eduardo Arce ${ }^{1}$, Sylvio Péllico Netto ${ }^{1}$, \\ Ana Paula Dalla Corte ${ }^{1}$, Ataides Marinheski Filho ${ }^{1}$, \\ Emanuel José Gomes de Araújo ${ }^{1}$
}

${ }^{1}$ Departamento de Engenharia Florestal, Universidade Federal do Paraná - UFPR, Curitiba/PR, Brasil

\section{RESUMO}

Foi conduzido um experimento em povoamentos de Pinus taeda com objetivo de avaliar o efeito do desbaste e da qualidade do sítio na relação hipsométrica. Efetuou-se uma análise de variância em blocos casualizados, em que os blocos corresponderam aos sítios e os tratamentos, aos desbastes mistos, combinando os métodos sistemático e seletivo baixo, cortando uma a cada cinco linhas de plantio mais 66\%, 54\%, 42\%, 30\% e 19\% de árvores do dossel inferior. Os desbastes ocorreram entre 8 e 9 anos de idade e, aos 12 anos, foram medidos o diâmetro e a altura das árvores remanescentes. A relação hipsométrica foi modelada e aplicado o teste de identidade de modelos de Graybill, para reduzir o número de modelos similares selecionados no mesmo bloco. Houve diferença estatística entre tratamentos e blocos. Concluiu-se que a hipsometria varia pouco entre tratamentos, mas reduz sua inclinação quando piora a qualidade do sítio. Equações hipsométricas iguais podem ser empregadas em diferentes tratamentos.

Palavras-chave: capacidade produtiva, desbaste misto, experimentação florestal, diâmetro, altura total.

\section{Thinning and Site Quality Effects on the Height-diameter Relationship of Pinus taeda}

\begin{abstract}
An experiment in pine (Pinus taeda) stands was conducted aiming to evaluate the thinning and site quality effect on the height-diameter relationship. We applied an analysis of variance in randomized blocks, with blocks representing the sites and treatments the mixed thinning, in which systematic and selective modalities were combined, harvesting every fifth row plus $66 \%, 54 \%$, $42 \%, 30 \%$ and $19 \%$ of the lower canopy trees. Thinnings occurred at 8 and 9 years old interval, and diameters and heights of the trees were measured at age 12 . We modeled height-diameter relationship and applied the Graybill test for reducing the number of similar selected models in a same block. We found statistical differences between treatments and blocks. As conclusion, height-diameter relationship varies less between treatments but the curve slope tends to reduce in lower quality sites. Same height-diameter equations can be used in different treatments.
\end{abstract}

Keywords: productive capacity, mixed thinning, forest experimentation, diameter, total height. 


\section{INTRODUÇÃO}

O aumento da demanda por madeira no mercado brasileiro induz os técnicos da área florestal a adotarem medidas que aumentam a produtividade da floresta, como o melhoramento genético e técnicas silviculturais adequadas, dentre as quais se pode mencionar a prática dos desbastes, os quais variam de acordo coma finalidade do manejo florestal.

Na produção de toras de grande diâmetro, visadas por serem de alto valor agregado, a aplicação de desbastes no povoamento é bastante comum, pois a retirada de algumas árvores estimula o crescimento radial das remanescentes. Além disso, a colheita proveniente do desbaste pode antecipar receita, em vez de ela ser obtida apenas no corte raso.

No sul do Brasil, comumente são aplicados em povoamentos de pinus desbastes do tipo por baixo (ou alemão), em que árvores do dossel inferior são removidas, e do tipo sistemático (ou mecânico), em que se removem linhas de plantio com intervalo preestabelecido (Smith et al., 1996).

Contudo, muitas vezes a escolha do tipo, idade e peso do desbaste é baseada em fundamentos empíricos, tornando-se a técnica dependente da experiência do manejador. Nesse sentido, as técnicas que auxiliam na Determinação da melhor opção de desbaste funcionam como uma importante ferramenta no manejo florestal (Nogueira et al., 2001; Finger \& Schneider, 1994, 1999).

Embora essas técnicas envolvam apenas métodos quantitativos e de estoque, a adoção do regime de desbaste deve levar em Consideração questões técnicas e econômicas, visto que cada alternativa apresenta peculiaridades específicas (Schneider \& Schneider, 2008).

Conforme Assmann (1961), a retirada de árvores altera as relações dendrométricas, visto que o procedimento consiste em uma intervenção ríspida na interação entre indivíduos do povoamento. Essas condições demandam que regimes de desbaste sejam executados de preferência com planejamento prévio.

Outro fator relevante para o manejo relaciona-se à capacidade de sítio, pois as condições edafoclimáticas exercem efeito no crescimento e na produtividade florestal, demonstrando que as variáveis dendrométricas são influenciadas tanto pela competição entre indivíduos como pelo sítio (Machado et al., 2008; Bartoszeck et al., 2004).

Nesse escopo, parte do conhecimento das boas práticas de manejo é alcançado com os estudos relacionados ao comportamento biométrico de povoamentos submetidos a diferentes regimes de desbaste e a diferentes sítios florestais.

Esta pesquisa teve como objetivo avaliar o efeito de 5 regimes de desbastes mistos (sistemáticos e seletivos baixos), realizados em 4 qualidades de sítio florestais, nas variáveis diâmetro a 1,3 m do solo (dap) e altura total (h), em povoamentos de Pinus taeda L. localizados no estado de Santa Catarina.

\section{MATERIAL E MÉTODOS}

\section{1. Área de estudo e experimento}

O experimento foi conduzido em povoamentos de Pinus taeda L. localizados nos municípios de Campo Alegre e Joinville, estado de Santa Catarina, cujo espaçamento de plantio foi de $2,5 \mathrm{~m} \times 2,5 \mathrm{~m}$, totalizando em 1.600 plantas por hectare. A área selecionada era composta por quatro talhões, os quais representaram quatro sítios florestais previamente classificados, adotando-se a idade índice de 15 anos (David, 2014). As alturas dominantes dos sítios I, II, III e IV equivaleram a 27,8 m, 23,3 m, 18,8 m e 14,3 m, respectivamente.

Para realizar a pesquisa foram utilizados dados de 3.544 árvores em 183 parcelas circulares de $201 \mathrm{~m}^{2}$, amostradas sistematicamente em um grid de $100 \mathrm{~m} \times 100 \mathrm{~m}$. O experimento consistiu em submeter tais parcelas a 5 regimes de desbaste, realizados entre 8 e 9 anos de idade.

Os desbastes foram do tipo misto, combinando o de tipo sistemático e o de tipo seletivo baixo (desbaste alemão), em que foram retiradas 1 a cada 5 linhas de plantio, bem como árvores escolhidas do estrato inferior do dossel, cuja variação de seleção diferenciou os 5 regimes adotados (Tabela 1 ).

Posteriormente, aos 12 anos de idade, as árvores remanescentes tiveram seu diâmetro a 1,3 $\mathrm{m}$ do solo (dap) medido com fita diamétrica, bem como sua altura total (h) medida com hipsômetro Vertex IV. Foram calculados os valores médios de dap e h em 
Tabela 1. Experimento florestal com parcelas de Pinus taeda em Santa Catarina.

Table 1. Forest experiment with Pinus taeda plots, in Santa Catarina state.

\begin{tabular}{|c|c|c|c|c|}
\hline \multirow{2}{*}{ Bloco } & \multirow{2}{*}{ Tratamento } & \multicolumn{3}{|c|}{ Desbaste } \\
\hline & & Remanescente & Colheita & Tipo (misto) \\
\hline \multirow{5}{*}{$1,2,3$ e 4} & 1 & 435 árvores.ha ${ }^{-1}$ & 1.165 árvores.ha ${ }^{-1}$ & $5^{\mathrm{a}}$ linha $+66 \%$ por baixo \\
\hline & 2 & 589 árvores.ha $^{-1}$ & 1.011 árvores.ha $^{-1}$ & $5^{\mathrm{a}}$ linha $+54 \%$ por baixo \\
\hline & 3 & 742 árvores.ha ${ }^{-1}$ & 858 árvores.ha ${ }^{-1}$ & $5^{\mathrm{a}}$ linha $+42 \%$ por baixo \\
\hline & 4 & 896 árvores.ha ${ }^{-1}$ & 704 árvores.ha $^{-1}$ & $5^{\mathrm{a}}$ linha $+30 \%$ por baixo \\
\hline & 5 & 1.037 árvores.ha $^{-1}$ & 563 árvores.ha ${ }^{-1}$ & $5^{\mathrm{a}}$ linha $+19 \%$ por baixo \\
\hline
\end{tabular}

cada sítio e regime de desbaste e, assim, feita uma análise estatística.

A comparação estatística foi feita por meio da análise de variância (ANOVA) com prévia constatação de homogeneidade das variâncias pelo teste de Bartlett. $\mathrm{O}$ experimento foi estruturado em delineamento em blocos ao acaso, em que os tratamentos corresponderam aos regimes de desbaste e os blocos, aos sítios florestais (Tabela 1).

Nos casos em que a ANOVA revelou diferença estatística ao nível de 5\% de significância realizou-se o teste de comparação de médias de Tukey, mantendo-se o mesmo nível de significância de 5\%. A finalidade foi avaliar o efeito da densidade de árvores e da qualidade do sítio florestal em ambas as variáveis.

\subsection{Modelagem hipsométrica}

Foram ajustados modelos hipsométricos, por tratamento e por bloco, totalizando 20 parcelas modeladas. Os modelos testados foram ajustados pelo método dos mínimos quadrados e estão apresentados na Tabela 2.

Foram testadas três estatísticas como critério de seleção do melhor modelo, obedecendo ao menor erro padrão da estimativa em porcentagem $\left(\mathrm{s}_{\mathrm{yx}} \%\right)$, maior coeficiente de determinação ajustado $\left(\mathrm{R}_{\mathrm{aj}}^{2}\right)$ e a melhor dispersão dos resíduos. As fórmulas para cálculo das estatísticas $\mathrm{s}_{\mathrm{yx}} \%$ e $\mathrm{R}_{\mathrm{aj}}^{2}$ p podem ser vistas em Araújo et al. (2012).

\subsection{Teste de identidade entre modelos}

Foi empregado o teste de identidade de modelos desenvolvido por Graybill (1976) para identificar a igualdade estatística entre as equações (Araújo et al., 2012). O teste permite empregar um menor número de equações ao agrupar tratamentos que tiveram os
Tabela 2. Modelos hipsométricos ajustados para parcelas de Pinus taeda aos 12 anos de idade, em Santa Catarina.

Table 2. Height-diameter models fitted to Pinus taeda plots with 12 years old, in Santa Catarina state.

\begin{tabular}{lc}
\multicolumn{1}{c}{ Autor } & \multicolumn{1}{c}{ Modelo } \\
Henricksen & $\mathrm{h}=\mathrm{a}_{0}+\mathrm{a}_{1} \cdot \ln (\mathrm{dap})+\varepsilon$ \\
Assmann & $\mathrm{h}=\mathrm{a}_{0}+\mathrm{a}_{1} \cdot\left(\frac{1}{\text { dap }}\right)+\varepsilon$ \\
Trorey & $\mathrm{h}=\mathrm{a}_{0}+\mathrm{a}_{1} \cdot \mathrm{dap}+\mathrm{a}_{2} \cdot \mathrm{dap}^{2}+\varepsilon$ \\
Curtis & $\ln (\mathrm{h})=\mathrm{a}_{0}+\mathrm{a}_{1} \cdot\left(\frac{1}{\mathrm{dap}}\right)+\varepsilon$ \\
Prodan & $\mathrm{h}=\frac{\mathrm{dap}^{2}}{\mathrm{a}_{0}+\mathrm{a}_{1} \cdot \mathrm{dap}^{\circ}+\mathrm{a}_{2} \cdot \text { dap }^{2}}+1,3+\varepsilon$ \\
Stoffels & $\ln (\mathrm{h})=\mathrm{a}_{0}+\mathrm{a}_{1} \cdot \ln (\mathrm{dap})+\varepsilon$
\end{tabular}

h: altura total, em m; dap: diâmetro a 1,3 $\mathrm{m}$ do solo, em $\mathrm{cm}$; $\mathrm{a}_{\mathrm{i}}$ : parâmetros do modelo; ln: logaritmo neperiano; $\varepsilon$ : erro aleatório

mesmos modelos selecionados. O agrupamento foi feito para tratamentos de mesmo bloco.

A técnica baseia-se na diferença da soma de quadrados da regressão (SQReg) dos modelos completos, ou seja, ajustados separadamente em cada tratamento, pela SQReg do modelo reduzido, ajustado com os dados dos tratamentos agrupados, por terem o mesmo modelo selecionado. Conforme Regazzi (1996), as hipóteses do teste são formuladas do seguinte modo:

- Hipótese inicial (H0): Os modelos completos são estatisticamente iguais ao modelo reduzido;

- Hipótese alternativa (H1): Nem todos os modelos completos são estatisticamente iguais ao modelo reduzido. 
Sob ambas as hipóteses, o valor de F de Graybill, quando não significativo, indica aceitação da hipótese inicial de igualdade entre os modelos, caso contrário, o teste indica que pelo menos um tratamento deve ser modelado separadamente aos demais. Assim foram feitas todas as combinações possíveis para avaliar qual(is) tratamento(s) deveria(m) ser isolado(s).

\section{RESULTADOS E DISCUSSÃO}

\subsection{Experimentação florestal}

O teste de Bartlett apontou homogeneidade das variâncias para as variáveis diâmetro (dap) e altura (h) analisadas, sendo observados valores de qui-quadrado corrigido $\left(\chi_{\text {corr }}^{2}\right)$ inferiores ao $\chi_{\text {tabelado }}^{2}=7,81$, ao nível de $5 \%$ de significância.

A análise de variância revelou que os tratamentos e os blocos exerceram efeito no dap e na h das árvores, ao nível de 5\% de significância. Logo, foi aplicado o teste de Tukey, nessa mesma probabilidade, para detectar tratamentos e blocos diferentes entre si. Os resultados, bem como outras estatísticas descritivas, estão apresentados na Tabela 3.

Os valores de $\mathrm{F}_{\text {calculado }}$ mostraram que os efeitos dos tratamentos e dos sítios foram altamente significativos sobre as variáveis. Resultados similares foram obtidos por Araújo et al. (2012) para Eremanthus erythropappus (DC.) MacLeish, por Inoue et al. (2011) para Pinus taeda, por Trevisan et al. (2007) para Eucalyptus grandis Hill ex Maiden, por Leite et al. (2006) também para
Pinus taeda e por Berger et al. (2002) para Eucalyptus saligna Smith.

Considerando os regimes de desbaste adotados, foi assumida uma condição de retirada de árvores do dossel inferior, o que propicia o estreitamento da distribuição normal das variáveis, proporcionalmente ao número de árvores desbastadas. Como consequência, os tratamentos foram compostos por indivíduos naturalmente maiores em $\mathrm{h}$, contribuindo para que seus valores médios fossem tão superiores quanto maior for o rigor do desbaste.

Do mesmo modo, as diferenças significativas nas médias de dap, em todos os tratamentos, foram influenciadas pela redução de árvores do dossel inferior e não apenas pelo efeito da densidade de árvores.

Em relação aos blocos, os resultados apresentados na Tabela 3 mostraram que a qualidade do sítio florestal também influencia na média do dap e, sobretudo, da $\mathrm{h}$, sendo isso confirmado por meio dos valores de $\mathrm{F}_{\text {calculado }}$. Resultados semelhantes foram encontrados por Machado et al. (2008) e Bartoszeck et al. (2004) para Mimosa scabrella (Benth.).

Adicionalmente, os valores de coeficiente de variação dos blocos (CV\%), em comparação ao dos tratamentos, foram superiores para todas as variáveis, indicando que o fator sítio florestal foi apropriadamente isolado por meio da análise em blocos ao acaso.

$\mathrm{O}$ maior valor observado de $\mathrm{F}_{\text {calculado }}$, correspondente à $\mathrm{h}$ na fonte de variação bloco, bem como sua diferenciação de todas as médias, confirma a alta correlação dessa

Tabela 3. Análises estatísticas e teste Tukey (5\% de significância) aplicados em parcelas experimentais de Pinus taeda, em Santa Catarina.

Table 3. Statistical analysis and Tukey test (5\% of significance) applied to experimental plots of Pinus taeda, in Santa Catarina state.

\begin{tabular}{|c|c|c|c|c|c|}
\hline Tratamento & dap $(\mathrm{cm})$ & $\mathbf{h}(\mathbf{m})$ & Bloco & $\operatorname{dap}(\mathrm{cm})$ & h (m) \\
\hline 1 & $24,7 \mathrm{a}$ & $17,4 \mathrm{a}$ & 1 & $27,8 \mathrm{a}$ & $22,4 \mathrm{a}$ \\
\hline 2 & $23,8 b$ & $17,2 \mathrm{~b}$ & 2 & $25,2 b$ & $18,9 b$ \\
\hline 3 & $23,2 \mathrm{c}$ & $17,1 b$ & 3 & $22,1 \mathrm{c}$ & $15,3 \mathrm{c}$ \\
\hline 4 & $22,8 \mathrm{~cd}$ & $16,9 c$ & 4 & $18,3 \mathrm{~d}$ & $11,7 \mathrm{~d}$ \\
\hline 5 & $22,2 d$ & $16,8 \mathrm{~d}$ & & & \\
\hline $\mathrm{F}_{\text {calculado }}$ & $56,83^{*}$ & $71,57^{\star}$ & $\mathrm{F}_{\text {calculado }}$ & $1.287,87^{*}$ & $30.379,81^{*}$ \\
\hline Média & 23,3 & 17,1 & Média & 23,3 & 17,1 \\
\hline $\mathrm{CV}(\%)$ & 4,1 & 1,5 & CV(\%) & 17,5 & 27 \\
\hline
\end{tabular}

Valores seguidos de mesma letra são estatisticamente iguais, ao nível de 5\% de significância, pelo teste de Tukey; dap: diâmetro a 1,3 m do solo; h: altura total; CV(\%): coeficiente de variação, em porcentagem; * significativo ao nível de $5 \%$ de significância. 
variável com os sítios, até mesmo para o dap, que foi estatisticamente diferente entre os blocos, ao nível de $5 \%$ de significância pelo teste de Tukey.

Esses resultados confirmam a importância de se classificar sítios florestais quanto à sua capacidade produtiva, pois mesmo em parcelas com diferentes densidades de árvores, o sítio comportou-se como um fator limitante ao crescimento diamétrico e da altura de árvores de Pinus taeda em Santa Catarina.

\subsection{Relações hipsométricas}

Os 6 modelos de relação hipsométrica ajustados aos 5 regimes de desbaste, nos 4 sítios, totalizaram 120 ajustes. Os resultados mostraram coeficientes significativos e não significativos ao nível de 5\% de significância, pelo teste $\mathrm{F}$.
Os valores do erro padrão da estimativa, em porcentagem s $s_{\mathrm{yx}}(\%)$, e do coeficiente de determinação ajustado $\left(\mathrm{R}_{\mathrm{aj}}^{2}\right)$, bem como dos coeficientes de ajuste dos modelos selecionados estão apresentados por parcela na Tabela 4.

A estatística de precisão $\mathrm{s}_{\mathrm{yx}} \%$ variou de $4,56 \%$ a 10,79\%, valores observados nas parcelas T4B2 e T2B4, respectivamente, e o $\mathrm{R}_{\text {aj }}^{2}$. esteve entre 0,2062 e 0,7842 , para as parcelas T2B4 e T1B1, respectivamente. Resultados semelhantes foram observados por Araújo et al. (2012) para Eremanthus erythropappus (DC), por Donadoni et al. (2010) para Pinus caribaea var. hondurensis e P. tecunumanii, por Soares et al. (2004) para Eucalyptus grandis W. Hill ex Maiden e por Bartoszeck et al. (2002) para Mimosa scabrella.

Em um contexto geral, os modelos de Curtis (4) e Prodan (5) apresentaram os melhores ajustes, sendo

Tabela 4. Coeficientes e estatísticas de ajuste e precisão de modelos de relação hipsométrica selecionados para parcelas experimentais de Pinus taeda, em Santa Catarina.

Table 4. Adjustment coefficients and statistics of height-diameter models selected for experimental plots of Pinus taeda, in Santa Catarina state.

\begin{tabular}{|ccrcrrrr|}
\hline Parcela & Autor & $\mathbf{a}_{\mathbf{0}}$ & $\mathbf{a}_{\mathbf{1}}$ & $\mathbf{a}_{\mathbf{2}}$ & $\mathbf{s}_{\mathbf{y x}}(\mathbf{\%})$ & $\mathbf{R}_{\text {aj }}^{\mathbf{*}}$ & $\mathbf{n}^{\mathbf{0}}$ \\
\hline T1B1 & Stoffels & $\mathbf{1 , 5 9 8 3 1}$ & $\mathbf{0 , 4 2 5 6 3}$ & & 5,34 & 0,7842 & 6 \\
\hline T2B1 & Curtis & $\mathbf{3 , 5 0 2 2 2}$ & $\mathbf{- 1 1 , 9 1 2 0 9}$ & & 6,61 & 0,7475 & 4 \\
\hline T3B1 & Henricksen & $\mathbf{- 1 2 , 8 4 5 4 5}$ & $\mathbf{1 0 , 1 9 7 0 8}$ & & 10,64 & 0,6609 & 1 \\
\hline T4B1 & Curtis & $\mathbf{3 , 4 2 0 3 5}$ & $\mathbf{- 1 0 , 6 7 2 2 2}$ & & 7,26 & 0,7415 & 4 \\
\hline T5B1 & Henricksen & $\mathbf{- 1 0 , 2 0 1 8 0}$ & $\mathbf{9 , 3 1 5 7 9}$ & & 7,36 & 0,7524 & 1 \\
\hline T1B2 & Stoffels & $\mathbf{1 , 9 9 4 4 5}$ & $\mathbf{0 , 2 8 3 0 9}$ & & 5,79 & 0,4912 & 6 \\
\hline T2B2 & Trorey & $\mathbf{6 , 6 9 0 7 3}$ & $\mathbf{0 , 6 6 8 8 1}$ & $\mathbf{- 0 , 0 0 8 1 3}$ & 6,74 & 0,5176 & 3 \\
\hline T3B2 & Curtis & $\mathbf{3 , 1 9 0 5 6}$ & $\mathbf{- 7 , 5 8 6 7 4}$ & & 5,32 & 0,6361 & 4 \\
\hline T4B2 & Prodan & 1,10073 & $\mathbf{0 , 3 7 5 9 5}$ & $\mathbf{0 , 0 4 3 0 0}$ & 4,56 & 0,7480 & 5 \\
\hline T5B2 & Prodan & $\mathbf{1 , 4 1 8 4 3}$ & $\mathbf{0 , 3 7 3 4 7}$ & $\mathbf{0 , 0 4 2 1 9}$ & 5,73 & 0,6872 & 5 \\
\hline T1B3 & Curtis & $\mathbf{3 , 1 4 9 3 6}$ & $\mathbf{- 8 , 1 3 1 6 3}$ & & 8,77 & 0,6667 & 4 \\
\hline T2B3 & Prodan & $\mathbf{6 , 9 8 6 9 7}$ & $-0,22393$ & $\mathbf{0 , 0 6 3 2 8}$ & 7,16 & 0,6356 & 5 \\
\hline T3B3 & Prodan & $\mathbf{3 , 0 9 8 7 5}$ & $\mathbf{0 , 2 7 7 3 1}$ & $\mathbf{0 , 0 4 9 0 3}$ & 7,31 & 0,6821 & 5 \\
\hline T4B3 & Curtis & $\mathbf{3 , 1 2 2 7 1}$ & $\mathbf{- 7 , 7 5 3 8 9}$ & & 6,02 & 0,7345 & 4 \\
\hline T5B3 & Prodan & $\mathbf{4 , 5 7 4 0 5}$ & 0,04808 & $\mathbf{0 , 0 5 5 9 4}$ & 6,23 & 0,7139 & 5 \\
\hline T1B4 & Henricksen & $-1,45876$ & $\mathbf{5 , 1 6 6 7 9}$ & & 8,23 & 0,4215 & 1 \\
\hline T2B4 & Assmann & $\mathbf{1 8 , 1 7 8 1 4}$ & $\mathbf{- 7 7 , 8 3 5 4 9}$ & & 10,79 & 0,2062 & 2 \\
\hline T3B4 & Assmann & $\mathbf{1 9 , 3 5 7 7 9}$ & $-\mathbf{1 0 8 , 0 3 2 4 3}$ & & 6,49 & 0,6281 & 2 \\
\hline T4B4 & Trorey & $\mathbf{- 0 , 4 2 8 1 6}$ & $\mathbf{1 , 2 2 5 7 5}$ & $-0,02472$ & 6,46 & 0,6688 & 3 \\
\hline T5B4 & Assmann & $\mathbf{1 8 , 1 1 7 4 3}$ & $\mathbf{- 8 3 , 6 2 5 7 1}$ & & 6,24 & 0,7192 & 2 \\
\hline
\end{tabular}

Letras T e B: tratamento e bloco, respectivamente; coeficientes em negrito são estatisticamente significativos pelo teste F de Fisher a $5 \%$ de significância; $\mathrm{a}_{\mathrm{i}}$ : parâmetros do modelo; $\mathrm{s}_{\mathrm{yx}} \%$ : erro padrão da estimativa, em porcentagem; $\mathrm{R}_{\mathrm{aj}}^{2}$ : coeficiente de determinação ajustado. 
cada um deles selecionado em 5 das 20 parcelas do experimento, seguidos dos modelos de Assmann (2) e Henricksen (1), ambos selecionados em 3 delas, e Stoffels (6) e Trorey (3), ambos selecionados em 2 parcelas.

Uma amostra dos resíduos da estimativa das alturas em porcentagem (R\%) pode ser observada na Figura 1. Os resíduos foram obtidos pelos modelos apresentados na Tabela 4. As letras T e B indicam tratamento e bloco, respectivamente.

Essa amostra representa os outros 16 gráficos, visto que eles mantiveram comportamento semelhante aos da Figura 1. As equações obtidas propiciaram estimativas não tendenciosas da altura em relação a uma escala de diâmetro a $1,3 \mathrm{~m}$ do solo (dap) de 0 a $50 \mathrm{~cm}$. Os resíduos estiveram majoritariamente contidos entre $\pm 25 \%$, em todas as parcelas do experimento.

As linhas de estimativa dos modelos hipsométricos apresentados na Tabela 4 podem ser observadas na Figura 2, por bloco (sítio florestal), em função do diâmetro a 1,3 $\mathrm{m}$ do solo (dap), em $\mathrm{cm}$. A letra T indica tratamento.

A Figura 2 confirma o comportamento esperado da altura total das árvores em sítios de diferentes capacidades produtivas, em que as linhas da estimativa tiveram sua inclinação reduzida do melhor (Figura 2a) para o pior sítio (Figura 2d), condizente com as alturas dominantes de $27,8 \mathrm{~m}$ e $14,3 \mathrm{~m}$, respectivamente.

O deslocamento das curvas para a direita do eixo das abscissas é resultado dos variados níveis de desbaste adotados, o que implica no crescimento diamétrico mais favorável para o tratamento 1 (menos denso), reduzindo-se para o tratamento 5 (mais denso).

No sítio I foi possível visualizar a distinção das linhas de estimativa das alturas a partir do diâmetro de $20 \mathrm{~cm}$, porém pouco pronunciada. Em relação ao sítio II, o comportamento da relação hipsométrica aparentemente foi mais homogêneo que nos demais sítios.
T1B1

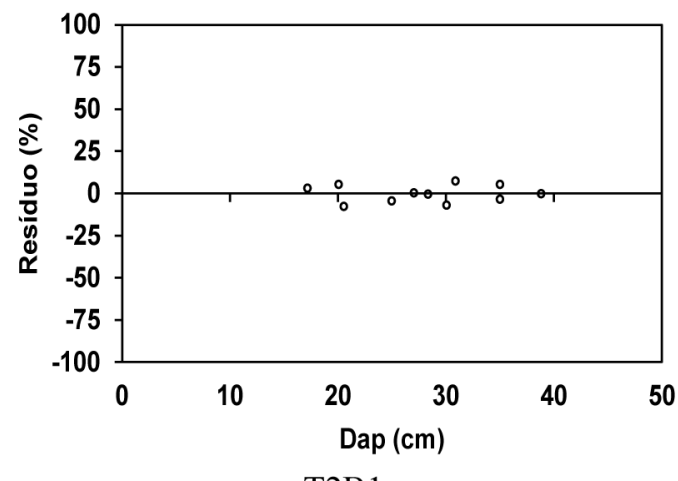

T2B1

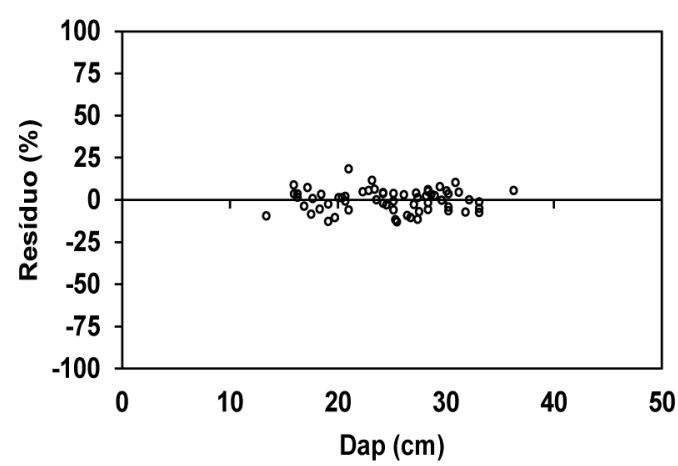

T1B2

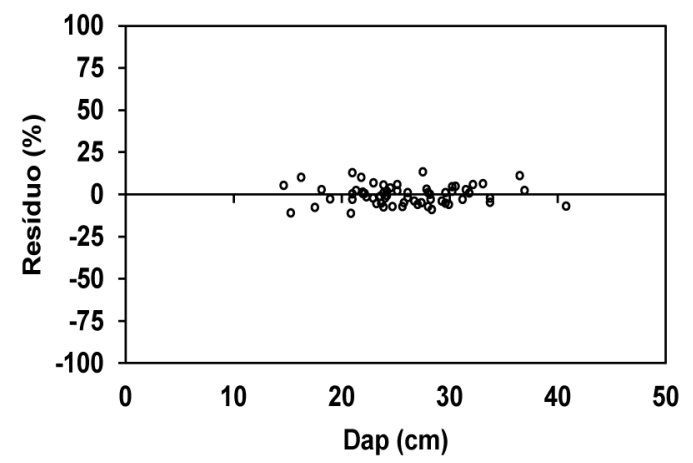

T2B2

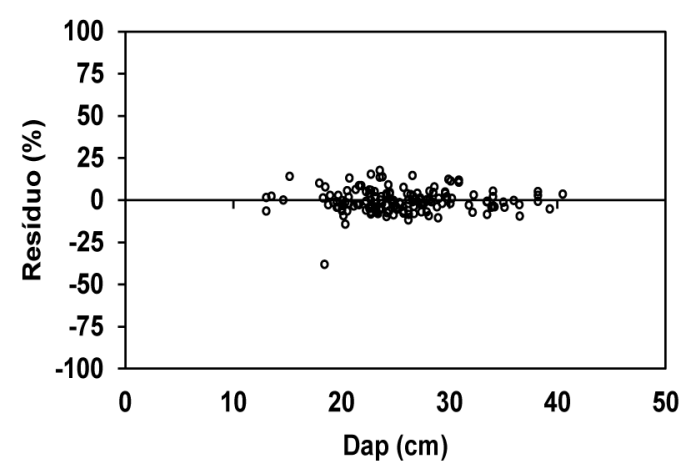

Figura 1. Dispersão de resíduos da estimativa de altura total para Pinus taeda em parcelas experimentais, em Santa Catarina.

Figure 1. Height estimative residuals scatter plots for Pinus taeda in experimental plots, in Santa Catarina state. 
$\longrightarrow \mathrm{T} 1---\mathrm{T} 2---\mathrm{T} 3$ T4 T5
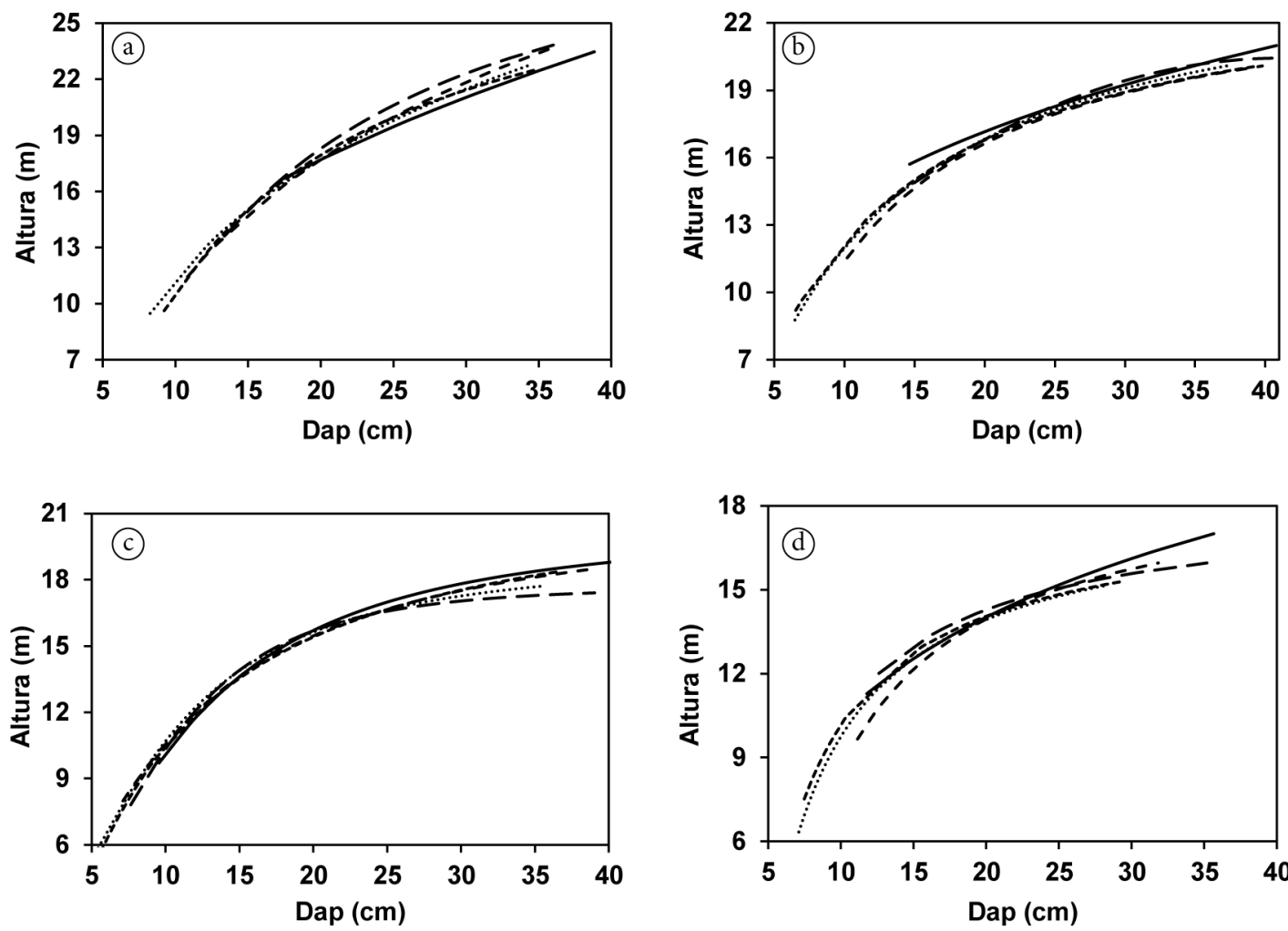

Figura 2. Altura estimada para Pinus taeda nos sítios I (a); II (b); III (c); e IV (d) em Santa Catarina.

Figure 2. Estimated heights for Pinus taeda on sites I (a); II (b); III (c); and IV (d) in Santa Catarina state.

Para os sítios III e IV, apesar de os tratamentos comportarem-se com certa equivalência, uma sutil diferença foi vista a partir do diâmetro de $30 \mathrm{~cm}$, com destaque para a curva do tratamento 1 , a qual se manteve acima das demais.

Os resultados mostram que o efeito do sítio florestal foi mais expressivo que a densidade de árvores e corroboram o observado por Bartoszeck et al. (2004) em povoamentos de Mimosa scabrella, em que o sítio e a idade afetaram mais a relação hipsométrica do que a densidade de árvores.

Em um estudo efetuado por Araújo et al. (2012), em plantios homogêneos de Eremanthus erythropappus, a partir da ilustração de gráficos de altura em função do dap, também foi possível notar comportamento semelhante da relação hipsométrica em quatro diferentes densidades de plantio.

\subsection{Identidade de modelos}

Com intuito de obter um menor número de equações por bloco (sítio), foi efetuado o teste de identidade entre modelos conforme proposto por Graybill (1976), o qual indicou semelhança entre equações em diferentes tratamentos no mesmo bloco, permitindo o emprego de uma mesma equação para tratamentos agrupados.

A Tabela 5 contém os resultados da análise de variância do teste $\mathrm{F}$ de Graybill. Os modelos reduzidos apresentaram coeficientes de ajuste significativos e não significativos pelo teste, ao nível de 5\% de significância.

Conforme a Tabela 5, tanto o erro padrão da estimativa em porcentagem $\left(\mathrm{S}_{\mathrm{yz}} \%\right)$ quanto o coeficiente de determinação ajustado $\left(\mathrm{R}_{\text {aj }}^{2}\right.$ ) tiveram valores próximos àqueles observados nos modelos completos. 
Tabela 5. Teste F de Graybill aplicado a modelos hipsométricos em parcelas experimentais de Pinus taeda, em Santa Catarina.

Table 5. F Graybill test applied to height-diameter models for Pinus taeda experimental plots, in Santa Catarina state.

\begin{tabular}{|c|c|c|c|c|c|c|c|c|}
\hline \multirow{2}{*}{ Parcelas agrupadas } & \multicolumn{6}{|c|}{ Modelo reduzido } & \multirow{2}{*}{$\mathbf{F}$} & \multirow{2}{*}{$\mathbf{H}_{0}$} \\
\hline & $\mathbf{N}^{\circ}$ & $a_{0}$ & $a_{1}$ & $a_{2}$ & $S_{y x} \%$ & $\mathbf{R}^{2}{ }_{a j}$ & & \\
\hline T2B1 e T4B1 & 4 & 3,45735 & $-11,19236$ & - & 7,08 & 0,7399 & $0,15^{\mathrm{ns}}$ & A \\
\hline T3B1 e T5B1 & 1 & $-10,96572$ & 9,56980 & - & 8,17 & 0,7310 & $0,20^{\text {ns }}$ & A \\
\hline T4B2 e T5B2 & 5 & 1,37936 & 0,36594 & 0,04270 & 5,37 & 0,7054 & $6,25^{*}$ & $\mathrm{R}$ \\
\hline T1B3 e T4B3 & 4 & 3,12802 & $-7,83159$ & - & 6,59 & 0,7197 & $0,18^{\text {ns }}$ & $\mathrm{A}$ \\
\hline Т2B3, Т3B3 е Т5B3 & 5 & 4,72105 & 0,04520 & 0,05588 & 6,71 & 0,6891 & $0,42^{\text {ns }}$ & A \\
\hline Т2B4, Т3В4 е Т5B4 & 2 & 18,52752 & $-90,03837$ & - & 7,47 & 0,5760 & $0,03^{\text {ns }}$ & A \\
\hline
\end{tabular}

Letras T e B, A e R: tratamento e bloco, aceitada e rejeitada, respectivamente; coeficientes em negrito são estatisticamente significativos pelo teste F de Fisher a 5\% de significância. ns: não significativo pelo teste F de Graybill a 5\% de significância; ${ }^{*}$ significativo pelo teste F de Graybill a 5\% de significância, respectivamente; $a_{\mathrm{i}}$ : parâmetros do modelo; $\mathrm{S}_{\mathrm{yx}} \%$ : erro padrão da estimativa, em porcentagem; $\mathrm{R}_{\mathrm{aj}}^{2}$ : coeficiente de determinação ajustado.

Conforme a Tabela 5, puderam ser agrupados dois pares de parcelas do bloco 1 (sítio I), um par e um trio do bloco 3 (sítio III) e um trio de parcelas do bloco 4 (sítio IV). A rejeição de $\mathrm{H}_{0}$, na tentativa de agrupamentos das parcelas T4B2 e T5B2, impediu o uso de um modelo reduzido para modelar conjuntamente a hipsometria de ambas. Sendo assim, o teste propiciou a redução de 20 para 13 equações hipsométricas, considerando-se as 20 parcelas experimentais.

\section{CONCLUSÕES}

O sítio florestal afeta significativamente as médias de diâmetro e altura de árvores de Pinus taeda. As curvas que representam a relação hipsométrica tendem a reduzir sua inclinação dos sítios mais produtivos para os menos produtivos.

As curvas hipsométricas não apresentam diferenças significativas em função dos desbastes. Em sítios florestais de capacidades produtivas semelhantes podem ser agrupadas modelos hipsométricos ajustados a parcelas de Pinus taeda submetidas a diferentes pesos de desbaste.

\section{STATUS DA SUBMISSÃO}

Recebido: 9 mar., 2014

Aceito: 22 dez., 2014

\section{AUTOR(ES) PARA CORRESPONDÊNCIA}

\section{Hassan Camil David}

Departamento de Engenharia Florestal, Universidade Federal do Paraná - UFPR, Av. Pref. Lothário Meissner, 632, CEP 80210-170, Curitiba, PR, Brasil

e-mail: hassancamil@gmail.com

\section{REFERÊNCIAS}

Araújo EJG, Pelissari AL, David HC, Scolforo JRS, Péllico S No, Morais VA. Relação hipsométrica para candeia (Eremanthus erythropappus) com diferentes espaçamentos de plantio em Minas Gerais, Brasil. Pesquisa Florestal Brasileira 2012; 32(71): 257-268. http://dx.doi. org/10.4336/2012.pfb.32.71.257.

Assmann E. Waldertragskunde. Muenchen: BLV Verlagsgessellschaft; 1961.

Bartoszeck ACPS, Machado SA, Figueiredo A Fo, Oliveira EB. Dinâmica da relação hipsométrica em função da idade, do sítio e da densidade inicial de povoamentos de bracatinga da região metropolitana de Curitiba, PR. Revista Árvore 2004; 28(4): 517-533. http://dx.doi.org/10.1590/ S0100-67622004000400006.

Bartoszeck ACPS, Machado SA, Figueiredo A Fo, Oliveira EB. Modelagem da relação hipsométrica para bracatingais da região metropolitana de Curitiba-PR. Revista Floresta 2002; 32(2): 189-204.

Berger R, Schneider PR, Finger CAG, Haselein CR. Efeito do espaçamento e da adubação no crescimento de 
um clone de Eucalyptus saligna Smith. Ciência Florestal 2002; 12(2): 75-87.

David HC. Avaliação de sítio, relações dendrométricas e otimização de regimes de manejo de Pinus taeda L. nos estados do Paraná e de Santa Catarina [dissertação]. Curitiba: Setor de Ciências Agrárias, Universidade Federal do Paraná; 2014.

Donadoni AX, Pelissari AL, Drescher R, Rosa GD. Relação hipsométrica para Pinus caribaea var. hondurensis e Pinus tecunumanii em povoamento homogêneo no Estado de Rondônia. Ciência Rural 2010; 40(12): 1499-2504. http:// dx.doi.org/10.1590/S0103-84782010001200010.

Finger CAG, Schneider PR. Determinação de regimes de desbaste para povoamentos de Pinus elliottii E. do Planalto Ocidental no estado de Santa Catarina. Ciência Florestal 1994; 4(1): 43-59.

Finger CAG, Schneider PR. Determinação do peso do desbaste para florestas de Eucalyptus grandis Hill ex Maiden, com base no índice de espaçamento relativo. Ciência Florestal 1999; 9(1): 79-87.

Graybill FA. Theory and application of the linear model. Belmont: Duxbury Press; 1976.

Inoue MT, Figueiredo A Fo, Rodrigo L. Influence of vital growth space on height and diameter of Pinus taeda L. Scientia Forestalis 2011; 39(91): 377-385.

Leite HG, Nogueira GS, Moreira AM. Efeito do espaçamento e da idade sobre variáveis de povoamentos de Pinus taeda
L. Revista Árvore 2006; 30(4): 603-612. http://dx.doi. org/10.1590/S0100-67622006000400013.

Machado AS, Bartoszeck ACPS, Figueiredo A Fo, OLIVEIRA EB. Efeito da densidade e do sítio sobre as curvas de distribuição diamétrica em Bracatingais Nativos (Mimosa Scabrellabenth.) da região metropolitana de Curitiba. Ambiência 2008; 4(1): 37-50.

Nogueira GS, Leite HG, Campos JCC, Souza AL, Couto L. Determinação da idade técnica de desbaste em plantações de eucalipto utilizando o método dos ingressos percentuais. Scientia Forestalis 2001; (59): 51-59.

Regazzi AJ. Teste para verificar a igualdade de modelos de regressão. Pesquisa Agropecuária Brasileira. 1996; (31): 1-17.

Schneider PR, Schneider PSP. Introdução ao manejo florestal. 2. ed. Santa Maria: FACOS-UFSM; 2008.

Smith DM, Larson BC, Kelty MJ, Ashton PMS. The practice of silviculture: applied forest ecology. 9. ed. John Wiley and Sons; 1996.

Soares TS, Scolforo JRS, Ferreira SO, Mello JM. Uso de diferentes alternativas para viabilizar a relação hipsométrica no povoamento florestal. Revista Árvore 2004; 28(6): 845 854. http://dx.doi.org/10.1590/S0100-67622004000600009.

Trevisan R, Haselein CR, Santini EJ, Schneider PR, Menezes LF. Efeito da intensidade de desbaste nas características dendrométricas e tecnológicas da madeira de Eucalyptus grandis. Ciência Florestal 2007; 17(4): 377-387. 